\title{
Robotics in Pancreatic Surgery
}

\author{
Yakup Kulu, Thilo Hackert*
}

\author{
* Corresponding author: \\ Thilo Hackert, MD, MBA \\ Dept. of General, Visceral and \\ Transplantation Surgery \\ University of Heidelberg \\ Im Neuenheimer Feld 110 \\ 69120 Heidelberg \\ Germany \\ Phone +496221565150 \\ Fax +496221565969 \\ Thilo.Hackert@med.uni-heidelberg.de
}

\section{Abbreviations:}

MIS - minimally-invasive surgery;

PD - pancreatico-duodenectomy;

DP - distal pancreatectomy.
Department of General, Visceral and Transplantation Surgery, University of Heidelberg, Germany

\section{ABSTRACT}

Robotic procedures have been implemented and become an important development in pancreatic surgery with an increasing acceptance worldwide. Nearly all types of pancreatic surgery have been performed robotically and especially standardized resections such as distal pancreatectomy and partial pancreatico-duodenectomy have gained importance despite a potentially long learning curve and increased procedure costs.

The present review outlines the past and current developments in robotic pancreatic surgery and summarizes the available literature and evidence on the respective procedures.

Key words: minimally invasive surgery, robotic surgery, pancreatectomy, learning curve best regards

\section{BACKGROUND}

Since the 1980s minimally-invasive surgery (MIS) has been introduced in various surgical disciplines, starting with gynecological operations and extending to other fields including visceral surgery to a greater extent in the 1990s (1).

After acceptance of "small" procedures like appendectomy or cholecystectomy, indications were extended and more complex procedures adopted this technique. The first MIS partial pancreatico-duodenectomy (PD) was performed in 1994 (2) demonstrating that this was feasible in highly specialized centers, however, no wide-spread acceptance occurred.

With certain latency and with utilization of the - now standardized - MIS approaches, robotic surgery became a field of research and growing interest. The first robotic procedures included neurosurgery, as the advantages of highly precise movements and preparation made this kind of surgery a suitable field of application (3).

After its establishment as a small start-up joint-venture between academic institutions and industry as well as the US army, in 1995 the company Intuitive Surgical (C) was founded and introduced the DaVinci ${ }^{\circledR}$ system as a robotic platform in 1999, receiving FDA approval for MIS procedures in 2000 and for MIS prostatectomy in 2001 - the most commonly performed robotic procedure today. Currently, Intuitive Surgical@ has achieved a nearly exclusive worldwide monopolistic market position and the $\mathrm{DaVinci}^{\circledR}$ system is by far the most 
commonly used device, since 2014 in the "Xi" version as the latest upgrade.

In 1997 the first robotic cholecystectomy was performed by Jacques Himpens in Belgium, in 1998 the first coronary heart procedure by Matthias Mohr in Leipzig, Germany (4). The first colorectal robotic procedures were performed in 2002 including a right colectomy and a sigmoid resection, both for benign indications (5). Parallel to this, upper Gl procedures were started, including Heller's myotomy in 2001, and esopgageal resections in 2002-2004 (6-9). Regarding pancreatic surgery, distal pancreatectomies and enucleations were performed as early as 2001 (10). Giulianotti reported the first robotic PD in 2001 as well (11), however, due to the complex reconstruction, very few surgeons tried to adopt this operation in the following years before larger patient series were published, mainly from the Pittsburgh center (12).

The timeline for all of these robotic procedures reflects a general phenomenon when new technologies are introduced in daily practice, i.e. starting such procedures in patients with benign diseases before extending to malignant indications. A problematic aspect in this context is the fact that the implementation of new procedures precedes the generation of evidence despite a potentially widespread adoption by a period of ten to fifteen years. A recent example for this problem is the implementation of MIS hysterectomy for early-stage cervical cancer in the US (13). Since the mid-2000s, MIS hysterectomy has been performed increasingly and has been regarded as the standard of care despite the lack of high-evidence studies on its surgical and especially oncological feasibility. As late as 2018 the first large randomized controlled trial (RCT) has addressed this and shown unfavorable oncological outcomes with a significant impairment of long-term survival for patients undergoing MIS hysterectomy vs. open hysterectomy (14). This underlines that the general adoption of any new procedure may never been done uncritically and that an early evaluation in high-quality studies is crucial not to harm patients. For any type of robotic surgery, there are mainly observational studies to date, yet, a large number of RCTs are planned or already recruiting that aim at the comparison of robotic vs. conventions MIS or open procedures (15). Consequently, more data on the safety and oncological feasibility are awaited within the next three to five years. The present review summarizes the currently available data on robotic procedures in pancreatic surgery.

\section{Distal pancreatectomy}

A standard distal pancreatectomy (DP) is an operation with a relatively low complexity due to the lack of a reconstructive phase and most centers have experience with conventional laparoscopic DP. DP is considered as a suitable procedure when a robotic program is launched and can preferably be performed patients with benign (i.e. cystic) or small and clearly resectable malignant lesions in the beginning of a center's experience. When proceeding to complex DP procedures (i.e. including portal vein or additional organ resection), this is certainly possible in experienced hands, however, it should always be critically evaluated if MIS DP actually may offer a benefit compared to an open approach for the respective patients.

The currently available evidence regarding robotic DP comprises mainly monocentric and retrospective series with a focus on perioperative outcomes, but data on oncological efficacy are sparse. Two meta-analyses published in 2016 and 2019 have summarized these studies $(16,17)$. Including nine (six retrospective and three prospective) monocentric studies in 2016, Memeo et al. came to the conclusion that perioperative outcomes were similar between conventional MIS DP and robotic DP (16). The authors discussed this as a potential learning curve effect as centers included patients during their initial robotic experiences. This conclusion implies the expectance of the authors that robotic DP should offer advantages over conventional DP which could be underestimated due to a nonoptimal performance during the learning curve. The more recent meta-analysis by Nui et al. conducted a three-arm comparison (open vs. conventional MIS vs. robotic DP) and included 16 retrospective series as well as one prospective study (17). It found no statistically significant differences between conventional MIS and robotic DP regarding blood loss and perioperative morbidity. With a longer operation time for robotic DP, there was a significantly higher rate of spleen preservation when intended compared to conventional MIS DP and a reduced overall rate of morbidity for the robotic procedures. A detailed look at the studies included in the mentioned meta-analyses shows that these are mostly retrospective series with only small numbers of patients which compared either conventional MIS vs. robotic DP (17-24), robotic vs. open DP $(25,26)$ or all of the three procedures (29-32). In summary, robotic DP had a longer operation time compared to both other approaches without relevant differences in perioperative morbidity, however, robotic DP was associated with increased costs. When compared to 
open DP both, conventional MIS as well as robotic DP, were characterized by commonly assumed advantages of minimally-invasive surgery, namely reduced blood loss, faster recovery of the patients and shorter hospital stay. As a special aspect, some studies only focused on the topic of spleen preservation and the potential advantage of a robotic approach over conventional MIS in this respect (33-35). The results remain unclear as the hypothetic advantage of the telemanipulator use (more precise preparation, better view) were observed and discussed in these three studies (33-35), whereas a prospective non-randomized trial by Butturini et al. failed to confirm this (19). One registry-based US study investigated the oncological long-term results of conventional MIS vs. robotic DP (36). Comparing 99 robotic vs. 605 conventional MIS DPs with a median follow-up time of 25 months resulted in similar outcomes.

\section{Partial pancreatico-duodenectomy}

Partial pancreatico-duodenectomy (PD) is the most challenging procedure regarding robotic pancreas surgery. Although the robotic technology with the possibilities of three-dimensional instrument movement and high-definition view facilitates MIS technique, tissue handling and manipulation during resection as well as reconstruction requires a high level of expertise. Especially during the learning curve, increased morbidity may occur and high conversion rates are possible. Currently, there are no RCTs on the outcomes of PD compared to open or conventional MIS PD. A number of mono-and multicenter observational studies show promising results with regard to morbidity, mortality and oncological results (37-41). The largest published comparative series from the US includes eight centers and 211 post learning-curve robotic vs. 817 open PD (37). The study showed that in clinical practice neither a high BMI nor a history of previous abdominal surgery represent contraindications to choose a robotic approach resulting in an overall conversion rate of $4.7 \%$. Furthermore, the robotic technique was used for challenging pancreatic anastomoses (soft tissue, small duct) without a trend to convert to an open procedure in this situation. Interestingly, the authors observed for malignant indications, that surgeons tended to prefer an open approach as $55 \%$ of open vs. $33 \%$ of robotic PDs were performed for cancerous lesions. This may well be explained by basic concerns regarding radicality of the robotic procedures, however, results remain unclear in this respect. The proportion of R1 resections was higher for the robotic group ( $50 \%$ vs. $33 \%$ ) whereas the number of retrieved lymph nodes favored the robotic resection ( $n=27.5$ vs. $n=19$ harvested lymph nodes) without results on long-term outcomes. Perioperative outcomes were comparable, especially clinically relevant POPF (robotic $13.8 \%$ vs. open $9.0 \%$ ). With a similar length of hospital stay (eight days), robotic patients were readmitted more frequently $(31 \%$ robotic PD vs. $24 \%$ open PD). These results show that robotic PD is feasible in specialized institutions and short-term results are comparable with open PD. A definitive evaluation especially regarding long-term oncological outcomes is not possible from these data. A recent systematic review (38) includes 11 non randomized studies comparing robotic and open PD. The number of robotic procedures in the underlying studies varies between $n=8-211$ whereas $n=8-817$ open procedures are compared resulting in $n=514$ robotic vs. $n=1263$ open PD. These results show significant differences in operation time (robotically $+1.5 \mathrm{~h}$ ) and blood loss (robotically $-200 \mathrm{ml}$ ) with similar transfusion rate. Looking at the subgroup of oncological patients, in this pooled data analysis, robotic PD showed a lower rate of R1 resections with a similar number of retrieved lymph nodes. The data favor robotic PD in terms of lower overall morbidity (especially surgical site infections) and faster postoperative mobilization of the patients. However, faster mobilization does not turn into shorter length of hospital stay. This parameter - one of the potentially most important advantages of all MIS procedures - is given with 7 - 16 days for robotic vs. 8 - 25 days for open PD. Considering the low methodological quality of the included studies these results have to be considered with caution as only retro- or prospective observational studies have been included and a certain bias of underreporting events especially during the learning curve of a new procedure is inherent.

Extended PD has been performed in only a few $\mathrm{c}$ enters worldwide to date $(40,41)$. Principally, such operations are possible using the robotic system including vascular venous as well as arterial reconstructions. Due to the very limited number of patients undergoing extended robotic PD reported so far it is not possible to give a valid estimation about potential advantages. In addition, it has to be considered that considerable morbidity (up to $80 \%$ ) and mortality rates (up to $14 \%$ ) may be associated with these approaches. Consequently, the impact of robotics in extended PD has to be considered very low at the present stage and further studies have to be awaited to give a valid evaluation. Similarly, there are no relevant studies on 
robotic total pancreatico-duodenectomy although this procedure can certainly be performed as a combination of PD and DP and potential advantages discussed for the latter procedures may be assumed.

\section{Enucleation and central pancreatectomy}

Parenchyma-sparing pancreatic resections including enucleation or central pancreatectomy for benign cystic lesions or neuroendocrine tumors can be performed robotically with a high precision due to the excellent visualization and the possibilities of meticulous technical preparation of the system. Depending on the localization of the respective finding, the approach including patient placement as well as positioning of trocars and instruments must be directed towards the pancreatic head or the pancreatic body according to the approaches for PD or DP, accordingly. A precise preoperative localization of the target lesion is crucial as an extensive inspection of the pancreas is difficult and time-consuming in robotic surgery and any kind of haptic feedback is lacking. In uncertain cases, intraoperative ultrasound may be required to find or confirm the lesion's localization and show a possible proximity to the main pancreatic duct. After clear definition of the lesion a precise and meticulous preparation under excellent viewing conditions is one of the important advantages that the robotic approach offers. Outcomes seem to be similar to open approaches without increased morbidity, especially POPF, when the main pancreatic duct is not approached and a distance of the lesion of at least $2 \mathrm{~mm}$ is respected (42-45). A potential advantage in terms of earlier patient discharge without increased rates of readmission may be achieved by robotic compared to open enucleation $(42,43)$

\section{Other procedures}

Other pancreatic procedures can basically be performed robotically or robotically assisted; however, such operations have been reported only anecdotally. Especially with regard to procedures in chronic pancreatitis - characterized by extensive inflammatory and fibrotic tissue changes as well as potentially pronounced porto-venous collateral flow - all types of MIS procedures have to be handled with care due to the challenging preparation and the increased risk of bleeding that may be difficult to control. Few case reports or case series have been published [i.e. for duodenum-preserving pancreatic head resection (46, 47)] and underline the feasibility of these operations in experienced centers. Despite this, these indications and procedures remain rare and high-level evidence studies are unlikely to be expected due to the low frequency of performance. Comparably, robotic pancreas transplantation has been described (48) in a small number of patients but the performance of this procedure is also certainly limited to very few and highly specialized center and will not gain widespread importance.

\section{CONCLUSION}

The introduction of robotic procedures in the last two decades has changed all fields of surgery including visceral and pancreatic surgery. Especially for DP which is today mostly performed by conventional MIS technique, the robotic approach has been introduced by many centers due to the more convenient approach. Robotic PD is increasingly performed but a considerable learning curve has to be taken into account for this complex type of surgery. Currently, several large observational studies confirm the feasibility of both procedures. They show that potential MIS advantages such as earlier mobilization or shorter hospital stay may be achieved by robotics as well. As no RCTs are available yet a valid comparison between robotic and open or conventional MIS procedures is not yet possible. Larger RCTs are currently being planned or already recruiting to create more evidence in the near future.

\section{Conflict of interest}

None to declare.

\section{REFERENCES}

1. Mack MJ. Minimally invasive and robotic surgery. JAMA. 2001;285: 568-572.

2. Gagner M, Pomp A. Laparoscopic pylorus-preserving pancreatoduodenectomy. Surg Endosc. 1994;8(5):408-10.

3. Kwoh YS, Hou J, Jonckheere EA, Hayati S. A robot with improved absolute positioning accuracy for CT guided stereotactic brain surgery. IEEE Trans Biomed Eng. 1988;35(2):153-60.

4. Lane T. A short history of robotic surgery. Ann R Coll Surg Engl. 2018;100(6 sup):5-7.

5. Weber PA, Merola S, Wasielewski A, Ballantyne GH. Teleroboticassisted laparoscopic right and sigmoid colectomies for benign disease. Dis Colon Rectum. 2002;45:1689-94; discussion 1695-96.

6. Melvin WS, Needleman BJ, Krause KR, Wolf RK, Michler RE, Ellison EC. Computer-assisted robotic heller myotomy: initial case report. J Laparoendosc Adv Surg Tech A. 2001;11(4):251-3.

7. Melvin WS, Needleman BJ, Krause KR, Schneider C, Wolf RK, Michler RE, et al. Computer-enhanced robotic telesurgery. Initial experience in foregut surgery. Surg Endosc. 2002;16: 1790-1792.

8. Horgan S, Berger RA, Elli EF, Espat NJ. Robotic-assisted minimally invasive transhiatal esophagectomy. Am Surg. 2003;69:624-626.

9. Kernstine KH, DeArmond DT, Karimi M, Van Natta TL, Campos JH, Yoder MR, Everett JE. The robotic, 2-stage, 3-field esophagolymphadenectomy. J Thorac Cardiovasc Surg. 2004;127: 
1847-1849.

10. Joyce D, Morris-Stiff G, Falk GA, El-Hayek K, Chalikonda S, Walsh RM. Robotic surgery of the pancreas. World J Gastroenterol. 2014; 20(40):14726-32.

11. Giulianotti PC, Coratti A, Angelini M, Sbrana F, Cecconi S, Balestracci $\mathrm{T}$, Caravaglios $\mathrm{G}$. Robotics in general surgery: personal experience in a large community hospital. Arch Surg. 2003;138(7):777-84.

12. Zureikat AH, Moser AJ, Boone BA, Bartlett DL, Zenati M, Zeh HJ 3rd. 250 robotic pancreatic resections: safety and feasibility. Ann Surg. 2013;258(4):554-9; discussion 559-62.

13. Melamed A, Rauh-Hain JA, Ramirez PT. Minimally Invasive Radical Hysterectomy for Cervical Cancer: When Adoption of a Novel Treatment Precedes Prospective, Randomized Evidence. J Clin Oncol. 2019;37(33):3069-3074.

14. Ramirez PT, Frumovitz M, Pareja R, Lopez A, Vieira M, Ribeiro R, et al. Minimally Invasive versus Abdominal Radical Hysterectomy for Cervical Cancer. N Engl J Med. 2018;379(20):1895-1904.

15. Kirchberg J, Weitz J. Evidence for robotic surgery in oncological visceral surgery. Chirurg. 2019;90(5):379-386.

16. Memeo R, Sangiuolo F, de Blasi V, Tzedakis S, Mutter D, Marescaux $\mathrm{J}$, et al. Robotic pancreaticoduodenectomy and distal pancreatectomy: State of the art. J Visc Surg. 2016;153(5):353-359.

17. Niu X, Yu B, Yao L, Tian J, Guo T, Ma S, et al. Comparison of surgical outcomes of robot-assisted laparoscopic distal pancreatectomy versus laparoscopic and open resections: A systematic review and meta-analysis. Asian J Surg. 2019;42(1):32-45.

18. Benizri El, Germain A, Ayav A, Bernard JL, Zarnegar R, Benchimol D, et al. Short-term perioperative outcomes after robot-assisted and laparoscopic distal pancreatectomy. J Robot Surg. 2014;8(2): 125-132.

19. Butturini G, Damoli I, Crepaz L, Malleo G, Marchegiani G, Daskalaki $D$, et al. A prospective non-randomised single-center study comparing laparoscopic and robotic distal pancreatectomy. Surg Endosc. 2015;29(11):3163-3170.

20. Goh BK, Chan CY, Soh HL, Lee SY, Cheow PC, Chow PK, et al. A comparison between robotic-assisted laparoscopic distal pancreatectomy versus laparoscopic distal pancreatectomy. Int J Med Robot 2017;13(1).

21. Ielpo B, Duran H, Diaz E, Fabra I, Caruso R, Malave L, et al. Robotic versus laparoscopic distal pancreatectomy: A comparative study of clinical outcomes and costs analysis. Int J Surg. 2017;48:300-304.

22. Liu R, Liu Q, Zhao ZM, Tan XL, Gao YX and Zhao GD. Robotic versus laparoscopic distal pancreatectomy: A propensity scorematched study. J Surg Oncol. 2017;116(4):461-469.

23. Lyman WB, Passeri M, Sastry A, Cochran A, lannitti DA, Vrochides $D$, et al. Robotic-assisted versus laparoscopic left pancreatectomy at a high-volume, minimally invasive center. Surg Endosc. 2019; 33(9):2991-3000.

24. Souche R, Herrero A, Bourel G, Chauvat J, Pirlet I, Guillon F, et al. Robotic versus laparoscopic distal pancreatectomy: a French prospective single-center experience and cost-effectiveness analysis. Surg Endosc. 2018;32(8):3562-3569.

25. Lai EC and Tang CN. Robotic distal pancreatectomy versus conventional laparoscopic distal pancreatectomy: a comparative study for short-term outcomes. Front Med. 2015;9(3):356-360.

26. Ielpo B, Caruso R, Duran H, Diaz E, Fabra I, Malave L, et al. Robotic versus standard open pancreatectomy: a propensity score-matched analysis comparison. Updates Surg. 2019;71(1):137-144.

27. Waters JA, Canal DF, Wiebke EA, Dumas RP, Beane JD, AguilarSaavedra JR, et al. Robotic distal pancreatectomy: cost effective? Surgery. 2010;148(4):814-823.

28. Duran H, lelpo B, Caruso R, Ferri V, Quijano Y, Diaz E, et al. Does robotic distal pancreatectomy surgery offer similar results as laparoscopic and open approach? A comparative study from a single medical center. Int J Med Robot. 2014;10(3):280-285

29. Lee SY, Allen PJ, Sadot E, D'Angelica MI, DeMatteo RP, Fong Y, et al. Distal pancreatectomy: a single institution's experience in open laparoscopic, and robotic approaches. J Am Coll Surg. 2015 220(1):18-27.

30. Xourafas D, Ashley SW and Clancy TE. Comparison of Perioperative Outcomes between Open, Laparoscopic, and Robotic Distal
Pancreatectomy: an Analysis of 1815 Patients from the ACS-NSQIP Procedure-Targeted Pancreatectomy Database. J Gastrointest Surg. 2017:21(9):1442-1452.

31. Fisher AV, Fernandes-Taylor S, Schumacher JR, Havlena JA, Wang $\mathrm{X}$, Lawson EH, et al. Analysis of 90-day cost for open versus minimally invasive distal pancreatectomy. HPB (Oxford). 2019; 21(1):60-66.

32. Rodriguez M, Memeo R, Leon P, Panaro F, Tzedakis S, Perotto O, et al. Which method of distal pancreatectomy is cost-effective among open, laparoscopic, or robotic surgery? Hepatobiliary Surg Nutr. 2018;7(5):345-352.

33. Kang CM, Kim DH, Lee WJ and Chi HS. Conventional laparoscopic and robot-assisted spleen-preserving pancreatectomy: does da Vinci have clinical advantages? Surg Endosc. 2011;25(6):2004-2009.

34. Eckhardt S, Schicker C, Maurer E, Fendrich V and Bartsch DK. Robotic-Assisted Approach Improves Vessel Preservation in Spleen-Preserving Distal Pancreatectomy. Dig Surg. 2016;33(5): 406-413.

35. Chen S, Zhan Q, Chen JZ, Jin JB, Deng XX, Chen H, et al. Robotic approach improves spleen-preserving rate and shortens postoperative hospital stay of laparoscopic distal pancreatectomy: a matched cohort study. Surg Endosc. 2015;29(12):3507-3518.

36. Raoof M, Nota C, Melstrom LG, Warner SG, Woo Y, Singh G, et al. Oncologic outcomes after robot-assisted versus laparoscopic distal pancreatectomy: Analysis of the National Cancer Database. J Surg Oncol. 2018:118(4):651-656.

37. Zureikat AH, Postlewait LM, Liu Y, Gillespie TW, Weber SM, Abbott $D E$, et al. A Multi-institutional Comparison of Perioperative Outcomes of Robotic and Open Pancreaticoduodenectomy. Ann Surg. 2016;264(4):640-649.

38. Zhao W, Liu C, Li S, Geng D, Feng Y and Sun M. Safety and efficacy for robot-assisted versus open pancreaticoduodenectomy and distal pancreatectomy: A systematic review and meta-analysis. Surg Oncol. 2018;27(3):468-478.

39. Zureikat AH, Beane JD, Zenati MS, Al Abbas Al, Boone BA, Moser AJ, et al. 500 Minimally Invasive Robotic Pancreatoduodenectomies: One Decade of Optimizing Performance. Ann Surg. 2019 Dec 5. doi: 10.1097/SLA.0000000000003550. (Epub ahead of print)

40. Kauffmann EF, Napoli N, Menonna F, Vistoli F, Amorese G, Campani $D$, et al. Robotic pancreatoduodenectomy with vascular resection. Langenbecks Arch Surg. 2016;401(8):1111-1122.

41. Giulianotti PC, Addeo P, Buchs NC, Ayloo SM and Bianco FM. Robotic extended pancreatectomy with vascular resection for locally advanced pancreatic tumors. Pancreas. 2011;40(8): 1264-1270.

42. Chong JU, Kang CM. Robotic Single-Site Plus One Port: Pancreas Enucleation. J Gastrointest Surg. 2019;23(7):1527-1528.

43. Jin JB, Qin K, Li H, Wu ZC, Zhan Q, Deng XX, et al. Robotic Enucleation for Benign or Borderline Tumours of the Pancreas: $A$ Retrospective Analysis and Comparison from a High-Volume Centre in Asia. World J Surg. 2016;40(12):3009-3020.

44. Liu R, Wakabayashi G, Palanivelu C, Tsung A, Yang K, Goh BKP, et al. International consensus statement on robotic pancreatic surgery. Hepatobiliary Surg Nutr. 2019;8(4):345-360.

45. Kabir T, Tan ZZX, Syn N, Chung AYF, Ooi LLPJ, Goh BKP. Minimallyinvasive versus open enucleation for pancreatic tumours: A propensity-score adjusted analysis. Ann Hepatobiliary Pancreat Surg. 2019;23(3):258-264.

46. Jiang $Y$, Jin JB, Zhan $Q$, Deng $X X$, Peng $\mathrm{CH}$ and Shen BY. Robotassisted duodenum-preserving pancreatic head resection with pancreaticogastrostomy for benign or premalignant pancreatic head lesions: a single-centre experience. Int J Med Robot. 2018; 14(4): e1903.

47. Hamad A, Zenati MS, Nguyen TK, Hogg ME, Zeh 3rd HJ and Zureikat AH. Safety and feasibility of the robotic platform in the management of surgical sequelae of chronic pancreatitis. Surg Endosc. 2018;32(2):1056-1065.

48. Boggi U, Signori S, Vistoli F, D'Imporzano S, Amorese G, Consani G, et al. Laparoscopic robot-assisted pancreas transplantation: first world experience. Transplantation. 2012;93(2):201-206. 